\title{
Diseño y manufactura de un prototipo de arado de vertedera para motocultor.
}

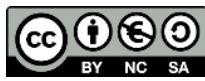

Design and manufacture of a prototype for motocultor moldboard plow.

Marcelo Castillo $^{1}$, Patricio Abarca ${ }^{2} \&$ David Bravo $^{3}$

Recibido: 09-12-2017 / Revisado: 05-02-2018 Aceptado: 12-03-2018/ Publicado: 01-04-2018

\begin{abstract}
.
DOI: https://doi.org/10.33262/cienciadigital.v2i2.78

The present work deals with the design and construction of: I) tools for mechanization of primary agriculture (farm tools) such as disc and mouldboard plows, furrowers and II) transportation tools for elements and inputs of agriculture: trailer and sprayer equipment and / or irrigation, both groups designed to be driven by a walking tractor; The objective of this written is to publicize the variety of tools that can be manufactured in the country, their uses and their impact on national agricultural production. In the design phase, data from ASABE (Society of Agronomists and Biologists) are used as the specific resistance values for the loads that can be assigned to the design software in accordance with the predominant deficient type in each ecuadorian region. INEN ISO 5718 Standard is also applied with respect to the minimum requirements of materials to be considered "agricultural" grade; Prototypes of implements have been obtained under Ecuadorian standards to which field trials have been carried out to validate their functionality; It is concluded that the prototypes collaborate in agricultural work, improve working conditions, save time and money in production and prevent diseases related to agriculture, finally make reference to the importance of the issue that the country is eminently agricultural and needs to be potentiated both the production of agro-parts and the improvement of working conditions in the rural sector, it is recommended to continue with the subsequent studies of the generated prototypes.
\end{abstract}

Keywords: Motocultor, Agricultural Implements, Specific.

\footnotetext{
${ }^{1}$ Escuela Superior Politécnica de Chimborazo, Ecuador, edison.castillo@espoch.edu.ec

${ }^{2}$ Escuela Superior Politécnica de Chimborazo, Ecuador, patricio.abarca@espoch.edu.ec

${ }^{3}$ Escuela Superior Politécnica de Chimborazo, Ecuador, david.bravo@espoch.edu.ec
} 


\section{Resumen.}

El presente trabajo trata del diseño y construcción de: I) herramientas para mecanización agrícola primaria (aperos) como: arados de disco y vertedera, surcadoras y II) herramientas de transporte para elementos e insumos del agro: remolque $\mathrm{y}$ equipo fumigador $\mathrm{y} / \mathrm{o}$ riego, ambos grupos diseñados para ser exclusivamente traccionados por motocultor; el objetivo del presente escrito es dar a conocer la variedad de aperos que se pueden fabricar en el país, sus usos y su impacto en la producción agrícola nacional. En la fase de diseño se usan datos de ASABE (Sociedad de Ingenieros Agrónomos y Biólogos por sus siglas en inglés) como son los valores de resistencia específica del suelo para analizar las cargas que se ha de asignar al software de diseño en concordancia con el tipo de suelo predominante en cada región ecuatoriana, también se aplica la Norma INEN ISO 5718 respecto de los requerimientos mínimos de los materiales para que sean considerados de grado "agrícola"; se han obtenido prototipos de aperos bajo normas ecuatorianas a los mismos que se han realizado ensayos de campo para validar su funcionalidad; se concluye que los prototipos ayudan significativamente en las labores agrícolas, mejoran las condiciones de trabajo, ahorran tiempo y dinero en la producción y previenen enfermedades profesionales relacionadas con la agricultura, finalmente cabe recalcar la importancia del tema ya que el país es eminentemente agrícola y se requiere potencializar tanto la producción de agropartes como de mejorar las condiciones de trabajo en el sector rural, se recomienda continuar con estudios posteriores de los prototipos generados.

Palabras Claves: Motocultor, Aperos, Resistencia Específica Del Suelo, Tipos De Suelo, Arado, Surcadora

\section{Introducción.}

En Ecuador y específicamente en la provincia de Chimborazo se desarrollaba una agricultura pobre en mecanización agrícola, producción mínima y de procesos de labrado de tierra artesanales, para los cuales se utilizaba la fuerza animal y mecanismos tradicionales de madera hechos por las mismas personas; sin estipular herramientas e implementos que faciliten el trabajo y hagan más productivas las labores del campo.

En la Facultad de Mecánica de la Escuela Superior Politécnica de Chimborazo, se han venido desarrollado tecnológicamente el diseño y construcción de los implementos para 
maquinaria agrícola e industrial con el afán de vincularse al país y mejorar el estilo de vida de los agricultores.

Con el diseño de implementos agrícolas que complementen el trabajo generado por el motocultor, se consigue aumentar la eficiencia de los procedimientos agrícolas para el arado de la tierra, debido a que estas técnicas disminuyen tiempo y mano de obra.

La utilización de grandes tractores provoca grandes problemas ocasionados por sus sistemas de arado que implican debido a su peso produciendo efectos negativos como es la compactación del suelo, además con un correcto equipamiento de los motocultores se evitará la adquisición de maquinaria pesada logrando disminuir el consumo de combustible y ahorrar espacio para el almacenamiento del motocultor y de aperos.

Cuando se adquiere un motocultor nuevo viene dotado de un único accesorio que ofrecen las casas comerciales de motocultores denominado "rotavator", el cual es costoso y no proporciona un eficaz trabajo agrícola. Este es un punto favorable para el desarrollo del presente proyecto, debido a que con el diseño de accesorios más adecuados aumentarán la diversificación de funciones a realizar y disminuirán los costos de producción agrícola.

El ministerio de agricultura, ganadería y pesca (MAGAP), regional de Chimborazo, cuenta con un número considerable de motocultores que han sido entregados dentro del país, sin embargo, no han podido ser utilizados debido a que no cuentan con los aperos apropiados para brindar un funcionamiento eficaz, produciendo un efecto negativo en el sector campesino en cuanto tiene que ver con la versatilidad de maquinaria y mejoramiento en la producción agrícola.

\section{Importancia del Problema.}

En el MAGAP de la provincia de Chimborazo existen alrededor de 150 motocultores YTO DF-15L acumulados en sus patios, que se importaron para suplir las necesidades de los agricultores chimboracenses, los que presentan baja fiabilidad en su órgano de labranza denominado "rotavator" o "rototiller", el cual presenta resistencia inferior a la que los terrenos de la provincia necesitan para ser labrados por tanto han provocado su rotura y a razón de estos quedan sin ser utilizados en busca de mejoras en sus implementos de laboreo del suelo.

Como resultado a esto, los agricultores no han adquirido la maquinaria, dado que incurriría en gastos de reparaciones e implementaciones, por lo tanto, los motocultores se encuentran en bodega por no tener más accesorios adecuados para el laboreo agrícola, provocando pérdidas económicas por almacenamiento y no contribuye al desarrollo agrícola. 


\section{Metodología.}

Conocidos también como tractores monoeje (un sólo eje de ruedas), son máquinas polivalentes que ofrecen grandes posibilidades en la agricultura minifundista (pequeñas explotaciones), en la agricultura de ocio, en los servicios municipales o terciarios y como complemento del tractor en la agricultura profesional latifundista, sobre todo en explotaciones hortofrutícolas y vitivinícolas (El Motocultor: Un Auxiliar Eficaz, 2009).

Gráfico 1. Motocultor

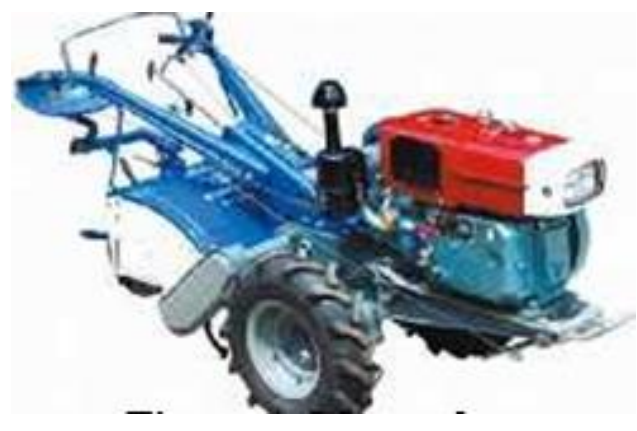

Fuente: Manual del motocultor YTO DF 15L

Consideraciones para el diseño del apero o herramienta de labranza.

\section{La ergonomía.}

Cuando tomamos en consideración los movimientos involucrados en la actividad diaria del operador y mejoramos su interactuación con la máquina en términos de eficacia, seguridad y confort, estamos hablando de la ergonomía.

\section{La seguridad.}

El motocultor es una máquina pionera en el trabajo de las labores agrícolas que en su dilatada vida ha permitido la incorporación de muchos sistemas de seguridad. Algunos de ellos han hecho variar inclusive la arquitectura de la máquina, dado que cualquier órgano en movimiento susceptible de herir al operario o a personas próximas se ha protegido mediante carenados, de forma que los elementos en movimiento no pueden alcanzar a personas ni proyectar elementos del terreno sobre éstas.

\section{Labranza del suelo.}

Labranza es la preparación del suelo para la siembra, y el proceso de mantenerlo suelto y libre de malezas durante el crecimiento del cultivo. La operación básica es la roturación del 
suelo en la preparación de la cama de siembra. La roturación y aflojamiento del suelo es la fase más antigua de la labranza.

Se estima que en la prehistoria el hombre usó herramientas rudimentarias hechas de madera u otro material con las cuales pudiera aflojar el suelo. Posiblemente una rama de árbol fue la primera herramienta de labranza usada por el hombre. Luego, con el uso del fuego o herramientas manuales de piedra, pudo el hombre modelar las ramas de los arboles con sus bifurcaciones, de manera que la herramienta de labranza poseía ya timón,

\section{Objetivos de la labranza.}

- Mezclar los residuos vegetales y animales mejorando la fertilidad al suelo.

- Enterrar y destruir malezas u otro tipo de vegetación indeseable.

- Descompactar el uelo para permitir una buena circulación de aire.

- Acondicionar el suelo para que retenga las aguas de lluvias.

- Cortar los ciclos de vida de los insectos, destruyendo sus huevos, larvas y nidos.

- Acondicionar la superficie del suelo para prevenir erosión eólica.

Los resultados de la aradura serán satisfactorios si el operador del arado sabe que condiciones de suelos se necesitan para el cultivo a implantar. El arado acondiciona una capa de suelo que constituye la cama de semillas y de raíces simultáneamente. Debemos recordar que el cultivo vive un $95 \%$ del tiempo en la cama de raíces y solo un $5 \%$ en la cama de semilla.

\section{Arado de reja y vertedera.}

El arado de reja y vertedera es uno de los más comunes. La reja penetra en la tierra y produce el corte de una porción de suelo que después es volteada con la vertedera, dejando abierto un surco en su recorrido.

Gráfico 2. Arado de Vertedera

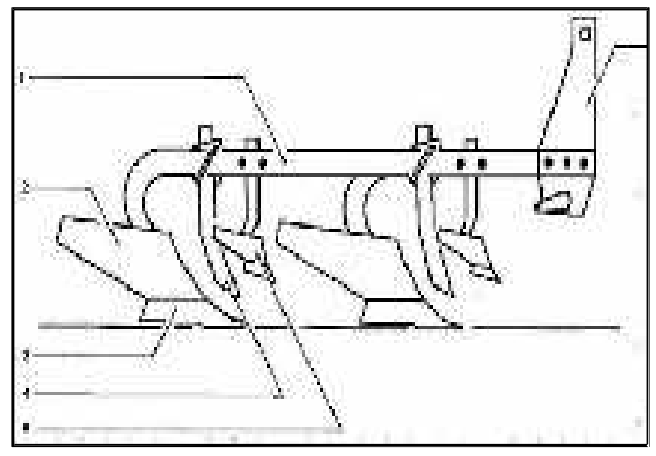

Fuente: Ortiz-Cañavate. 2012 


\section{Elementos de trabajo de los arados de reja y vertedera.}

Los términos usados corrientemente para denominar los principales componentes del arado son muy variados, de manera que daremos a continuación los que son generalmente aceptados.

- Cuerpo. - Se aplica al conjunto completo de piezas de trabajo para labrar un surco. Los cuerpos de los arados pueden ser de uso general, para labores profundas, etc.

- Reja. - Es la pieza que realiza el corte horizontal del prisma de tierra y lo separa del suelo que queda por debajo. Está montada en la parte delantera del cuerpo.

- Vertedera. - Es una lámina de metal sujeta con tornillos y convexa en toda su longitud que tiene como función voltear el pan o prisma de tierra.

- Dental. - Es la pieza que recibe el empuje lateral debido al volteo del pan o prisma de tierra.

- Cuchilla. - Realiza un corte vertical separando el prisma de tierra de la parte que no queda labrada en ese pase.

En muchos arados se coloca una cuchilla circular (disco) en posición vertical delante del cuerpo del arado, la cual cumple con la función de realizar un corte vertical que favorece el corte de la cuchilla, sobre todo en suelos pesados. (ECU-RED, 2016)

\section{Diseño del implemento surcador.}

Se procedió a utilizar la plataforma de modelado en 3D mediante software commercial.

Gráfico 3. Modelación en conjunto

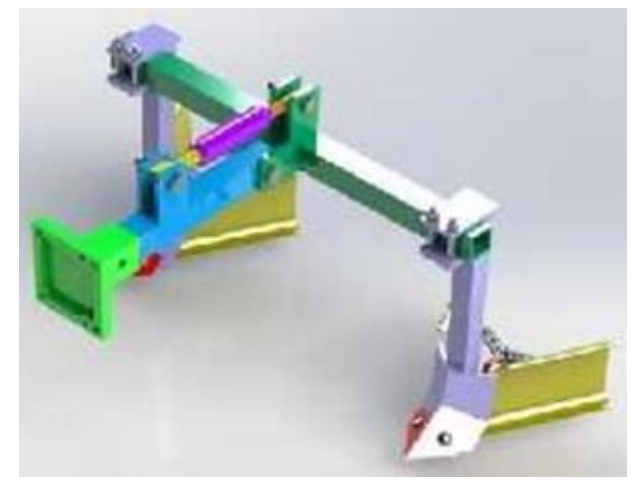

Fuente: Autores

Análisis de esfuerzos del implemento surcador.

Cargas verticales de diseño. 
Se efectúa un estudio de las cargas y esfuerzos a los que estará sometido el implemento surcador de forma vertical.

\section{Carga Permanente.}

Para determinar la carga permanente total $(\mathrm{Cp})$ del prototipo se toma los datos del peso propio del motocultor tomados del manual del motocultor (AINGLA, y otros, 2012).

El peso de todos los elementos del implemento surcador es de 41,238 kgf.

La carga principal que se ha de considerar es la del banco de tierra la cual va a ser labrada, para suelos medianos se tiene una Resistencia media de $50 \mathrm{kPa}$ y para los suelos típicos del centro del Ecuador 80kPa (Ortiz-Cañavate 2012)

\section{Simulación del implemento de labranza}

Para realizar una simulación de un proyecto cualquiera se necesita saber que plataforma se va utilizar y conocer el proceso de la simulación. En el caso del implemento surcador, se eligió la plataforma de simulación multifísica mediante el Método de Elementos Finitos, ANSYS.

\section{Resultados}

Gráfico 4. Modelación bajo carga

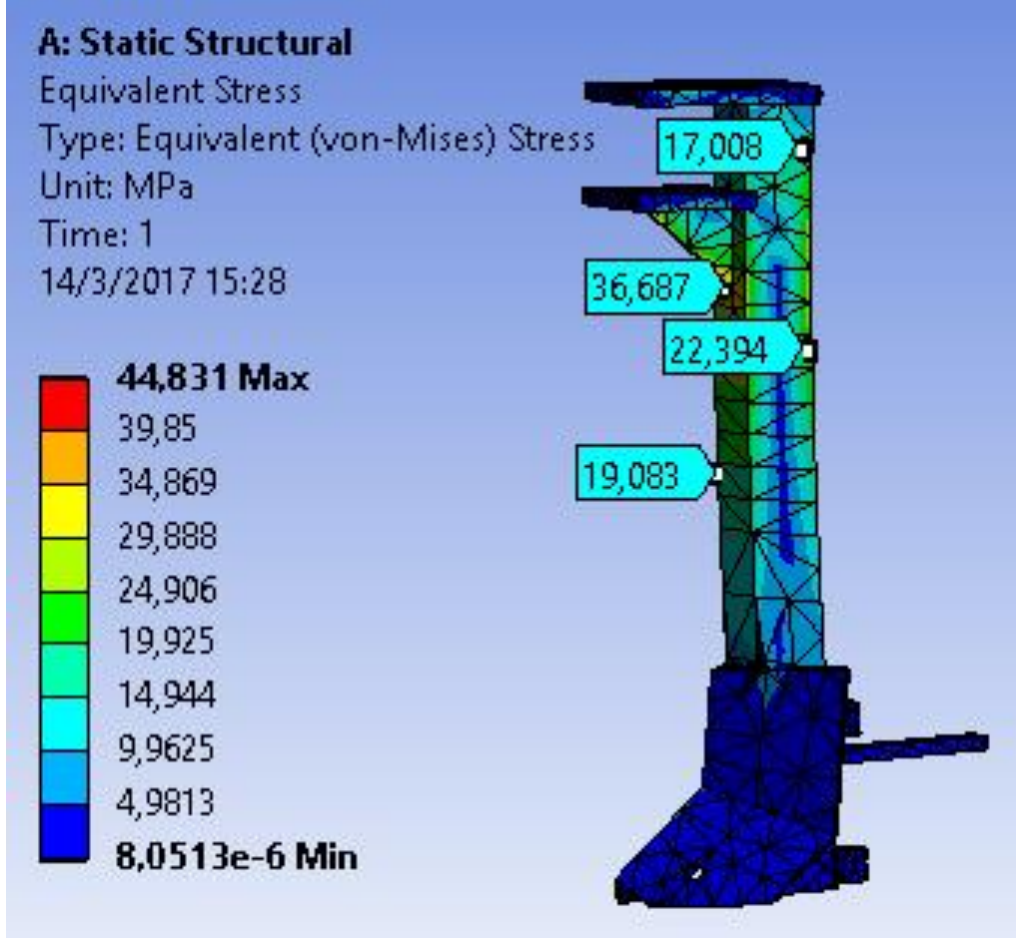

Fuente: Autores, 2018 


\section{Resultados de la simulación del implemento.}

Se necesita realizar la simulación de todos los elementos debido a las siguientes características que se mencionan a continuación: holgura entre elementos mecánicos, esfuerzos combinados, analizar las situaciones armónicas de los elementos, verificar la deformación total del implemento.

Se puede observar una deformación total de implemento de $7,33 \mathrm{~mm}$, este resultado se da por la holgura que existe entre los elementos, además su puede evidenciar que el implemento tiene un factor de seguridad total a fatiga de 1,24; cabe recalcar que los cálculos realizados anteriormente fueron realizados para un suelo franco. Las cuchillas de arado que fueron diseñadas con acero AIS I 1018 y FORA 450 (AISI 1024), no presentan inconveniente alguno, ya que en la simulación no se muestran deformaciones, además el esfuerzo equivalente mostrado en la simulación es muy inferior a la resistencia de cada cuchilla, con estas consideraciones finales se procedió a la construcción del implemento.

Cabe recalcar que se tomó una cuchilla con un material adicional al planificado con la finalidad de realizar un seguimiento al proyecto para verificar la durabilidad de los materiales a mediano y largo plazo.

\section{Discusión.}

El diseño y construcción de los elementos agrícolas destinados a la labranza primaria (arado) han de estar bajo consideraciones universalmente conocidas por ingenieros destinados a esta labor, por consiguiente, se detallan los parámetros a ser observados en el diseño y fabricación de aperos agrícolas:

- Deformación

- Factor de seguridad

- Dureza del material directo de labranza

En tal virtud la deformación total del bastidor del elemento de labranza se encuentra dentro del rango permisible para considerarse, es por ello que en el código de colores del software la máxima deformación está enmarcada en un color verde, muy lejos de la carga peligrosa que se considera cuando esté en color naranja y a su vez inadmisible si está bajo un color rojo en donde se considera que el elementos llegaría a su límite de fluencia.

Al considerar que la maquinaría agrícola está entre las más peligrosas y que el índice de accidentabilidad es alto, como también la tasa de mortalidad es considerable, un factor importante para tratar de reducir estos índices negativos es mejorar el factor de seguridad en el diseño, muchos autores podrían considerar que se está sobredimensionando 
elementos, pero es preferible trabajar con elementos mecánicos más robustos que correr el riesgo de ruptura en campo.

Por último es importante considerar que los elementos mecánicos que han de trabajar en mecanización agraria estarán sometidos a continuo desgaste, sobre todo en los suelos en donde la presencia de arcilla es mayor, por consiguiente se necesita materiales que resistan la abrasión y el desgaste, para ello se necesita que los materiales usados en la construcción del apero han de ser de "grado agrícola", lo que significa que su dureza superficial ha de estar entre el rango 40-50HRC, según los análisis desarrollados en los laboratorios se conoce que la herramienta de labranza cuenta con esa denominación.

\section{Conclusiones.}

- Se diseñó un implemento surcador de tierras que va a ser utilizado en las parcelas agrícolas de la provincia de Chimborazo, a través de la donación del Ministerio de Agricultura Ganadería Acuacultura y Pesca (MAGAP), esto servirá de ayuda a las personas beneficiadas mejorando el estándar de vida, también dando realce $\mathrm{y}$ aumento a la producción de alimentos verdes, para llevarlos al comercio local y/o nacional.

- Se procedió a construir el implemento, utilizando los materiales especificados en el proyecto, como son: ASTM A36, AISI 1018, FORA 450, los cuales se pueden adquirir en casas comerciales de metal dentro del país, estos materiales proveen de mucha confiabilidad al ser estructurales y según su contenido de carbono se ha implementado dos tipos de cuchillas en el mecanismo reja - vertedera

\section{Referencias bibliográficas.}

AINGLA, R \& QUIROZ, J. Diseño y construcción de un equipo de labranza con tracción accionada poor un motor de combustion interna para el tallado de un surco de siembra a la vez. Quito-Ecuador: Escuela Politécnica Nacional, 2012. pp 42-43

CAÑAVATE J. O. Las Máquinas Agrícolas y su Aplicación. Madrid-España : Mundi Prensa, 2012. pp 17-22.

ECU-RED. 2016. Arado. [En línea] Ecu - Red, Cuba: 2016. [Consultado: 13 de Diciembre de 2016] Disponible en: https://www.ecured.cu/Arado

LABOREO. "El Motocultor: Un Auxiliar Eficaz”. Laboreo, [En línea] 2009, España, Vol. 480, pp 54-57. [Consultado: 13 de Diciembre de 2016] . ISSN: 0210-1718. Disponible en: http://www.bcsagricola.com/ assets/blog/bcs/prensa/2009_11_motocultor_E SP_LATAM/articulo_bcs_laboreo_n480_novi embre09.pdf 
ECU-RED. 2016. Arado. [En lineal] Ecu - Red, Cuba: 2016. [Consultado: 13 de Diciembre de 2016] Disponible en: https://www.ecured.cu/Arado

CAÑAVATE J. O. El Tractor Agrícola, Madrid-España : Mundi Prensa, 2014. 


\section{Para citar el artículo indexado.}

Castillo M., Abarca P. \& Bravo T. (2018). Diseño y manufactura de un prototipo de arado de vertedera para motocultor. Revista electrónica Ciencia Digital 2(2), 145-154. Recuperado desde: http://cienciadigital.org/revistacienciadigital2/index.php/CienciaDigital/article/view/78/73

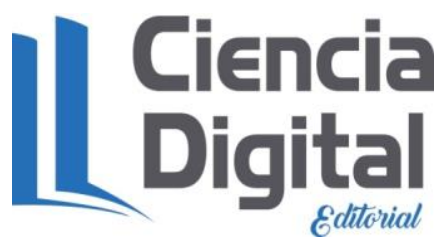

El artículo que se publica es de exclusiva responsabilidad de los autores y no necesariamente reflejan el pensamiento de la Revista Ciencia Digital.

El articulo queda en propiedad de la revista y, por tanto, su publicación parcial y/o total en otro medio tiene que ser autorizado por el director de la Revista Ciencia Digital.
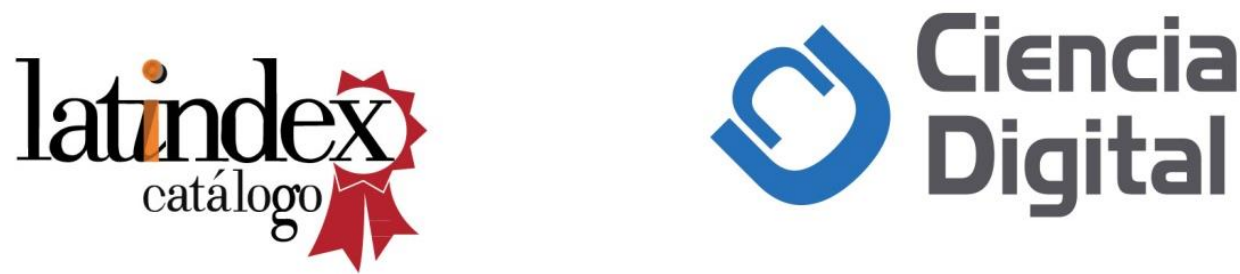